\title{
Introduction to a New Open Access Journal by MDPI: Future Physics
}

\author{
Edward K. Sarkisyan-Grinbaum ${ }^{1,2}$ \\ 1 Experimental Physics Department, CERN, 1211 Geneva 23, Switzerland; \\ Edward.Sarkisyan-Grinbaum@cern.ch \\ 2 Department of Physics, The University of Texas at Arlington, Arlington, TX 76019, USA
}

Received: 31 July 2018; Accepted: 31 July 2018; Published: 1 August 2018

check for updates

I am proud to announce the launch of a new MDPI journal, Future Physics, for which I am honored to serve as Editor-in-Chief as this topic is currently of high interest and it promises to be a very interesting publication forum.

Physics plays a crucial role in our understanding of the world. This already started when Aristotle divided all sciences into physics and metaphysics and, since that time, physics has contributed enormously to our progress and plays a great role in the development of other sciences moving forward. It is enough to recall just a few examples: the ideas of ADN coding in biology and the Big Bang for cosmology suggested by George Gamow; valence in chemistry to explain the periodic table of Dmitry Mendeleev; electromagnetic experiments by Michael Faraday; the equation of electromagnetism by James Maxwell; and differential calculus by Isaac Newton. These have revolutionized our understanding of nature, reaching new horizons in science and allowing us to make big steps towards progressing in each field. From Aristotle to Isaac Newton and then to Albert Einstein up to the present time, physics is in the center of human interest and irrevocably contributes to our knowledge moving into the future. This is confirmed again and again from century to century, and, most recently, by discoveries in particle physics and astrophysics, the latter breakthrough being the detection of gravitational waves, a triumph of Albert Einstein's general theory of relativity.

Considering all this, showing the huge significance of physics, it is timely to launch a journal dedicated to looking into future physics and in this sense connecting different branches and showcasing a diversity of topics under one umbrella.

I thank the Members of the Editorial Board for agreeing to serve on this new journal and invite them to contribute with their researches, as well as inviting papers in order to build a high scientific quality publication which will look for new horizons and discoveries in all areas of the modern physics movement and commence the ambitious task of clarifying contours of future physics.

(C) 2018 by the author. Licensee MDPI, Basel, Switzerland. This article is an open access article distributed under the terms and conditions of the Creative Commons Attribution (CC BY) license (http:/ / creativecommons.org/licenses/by/4.0/). 